\title{
Les recherches de Theodor Kocher sur l'étiologie de l'ostéomyélite et de la strumite aiguës
}

par Lazare Benaroyo

\section{RÉSUMÉ}

Theodor Kocher démontre expérimentalement en 1879 que l'ostéomyélite aiguë est une maladie infectieuse causée par un microorganisme non spécifique, le microcoque. Il montre que l'infection de la moelle osseuse est consécutive à une dissémination hématogène d'une infection locale, cutanée ou muqueuse. La forme clinique et pathologique que prend la maladie dépend, selon lui, non seulement de la virulence du microcoque, mais également de la qualité du terrain sur lequel ce dernier se développe. Il suggère la même année que la strumite aiguë (l'inflammation du goitre) a, par analogie, la même étiologie que l'ostéomyélite; il ne fournit cependant pas de preuves expérimentales à l'appui de cette hypothèse. Nous pouvons ainsi constater qu'à l'aube des années 1880, Kocher a défini de manière originale l'infection comme le produit d'une interaction entre un microorganisme et son hôte. L'influence exercée par ses idées sur ses contemporains atteste qu'il a stimulé les recherches sur l'étiologie des maladies infectieuses.

\section{L'ostéomyélite}

Si l'on injecte dans la moelle osseuse d'un chien de laboratoire, en prenant toutes précautions d'antisepsie nécessaires, des substances caustiques ou vésicantes $^{1}$, aucune inflammation ni aucune nécrose n'apparaissent; une légère sclérose circonscrite de l'os peut être tout au plus décrite.

Si l'on injecte par contre dans cette même moelle une petite quantité de liquide putride contenant des microcoques ${ }^{2}$, il se produit une ostéomyélite aiguë foudroyante entrainant le plus souvent la mort de l'animal par septicémie; une nécrose totale de l'os ainsi que des articulations avoisinantes est habituellement constatée dans ce cas à l'autopsie.

Si l'on introduit enfin dans l'alimentation de l'animal ce même microorganisme pathogène, plusieurs jours après avoir irrité sa moelle osseuse avec 
des substances chimiques, on peut observer le développement d'une ostéomyélite à l'endroit où a eu lieu l'irritation.

Telles sont les trois séries d'expérimentations rapportées par Theodor Kocher (1841-1917) - alors Professeur de chirurgie à Berne - dans un article paru en 1879 dans la revue Archiv für klinische Chirurgie ${ }^{3}$. Il y relate une conférence qu'il avait donnée le 10 avril 1878 au $7^{\text {ème }}$ Congrès de la Société allemande de Chirurgie, qui se tenait alors à Berlin. Mis à part ses expérimentations, Kocher rapporte dans cet article une longue série d'observations clinico-pathologiques ayant duré 6 ans, portant sur 26 cas, âgés de 8 à 25 ans. Il en publie le détail la même année dans la Deutsche Zeitschrift für Chirur$g e^{4}$. C'est à partir de ses expérimentations ainsi que de ses observations qu'il tire les conclusions suivantes:

1. L'ostéomyélite aiguë est une inflammation de la moelle osseuse très probablement consécutive à la présence de microcoques. Il s'agit d'une forme de microorganismes que son maître viennois, Theodor Billroth (18291894), avait déjà observée dans les inflammations des plaies ainsi que dans les abcès sous-cutanés. Billroth avait considéré que le microcoque était l'une des formes végétatives d'une algue microscopique qu'il avait dénommée Coccobacteria septica ${ }^{5}$. Kocher s'accorde à cette dénomination et considère de ce fait que le microcoque est un agent infectieux ubiquitaire qui n'est pas spécifique de l'ostéomyélite ${ }^{6}$.

2. Le microcoque peut parvenir au niveau de la moelle osseuse par voie hématogène; la porte d'entrée est alors le plus souvent digestive, lieu où il est habituellement présent lors d'infections respiratoires. Une porte d'entrée pulmonaire ou cutanée est également envisageable, même s'il n'y a plus aucune trace d'inflammation à ce niveau au moment où se développe l'ostéomyélite.

3. Le microorganisme observé lors de l'infection médullaire n'est pas le facteur causal exclusif de la maladie: le terrain joue également un rôle important. Le microorganisme exprime au mieux sa virulence au lieu de moindre résistance; c'est ainsi que l'ostéomyélite survient de préférence si l'os a auparavant subi un traumatisme ou un refroidissement. Ces deux circonstances produisent en effet des troubles circulatoires qui peuvent rendre, aux yeux de Kocher, le terrain plus fragile. De même une zone de croissance épiphysaire est-elle chez l'enfant un lieu de prédilection pour le développement d'une infection de la moelle osseuse.

4. Les différents tableaux cliniques allant de la simple plaie infectée localisée à la pyémie en passant par l'ostéomyélite aiguë, ne sont en fait, 
selon le chirurgien bernois, que les divers stades ou les divers degrés d'une même inflammation infectieuse microbienne. La différence entre une plaie, une ostéomyélite et une pyémie n'est en fait, du point de vue étiologique, qu'une différence quantitative. Cette différence est justement due à la qualité du terrain: un lieu de moindre résistance occasionne la survenue de métastases qui reflètent la gravité de l'infection de l'organisme.

Kocher est tenté de généraliser ce principe à toute forme d'inflammation infectieuse.

Ces quatre conclusions apportent les preuves expérimentales répondant de manière convaincante à la question qui préoccupait ses collègues chirurgiens et pathologues de l'époque: si la pyémie semble bien être, comme l'affirme dès 1872 Edwin Klebs ${ }^{7}$, une dissémination hématogène des microorganismes présents dans les plaies superficielles, peut-on admettre par analogie que l'ostéomyélite aiguë est un cas particulier de ce processus? Cette affection correspondrait dans ce cas à la métastatisation profonde d'un microorganisme ayant pénétré par la peau ou par une muqueuse. Kocher démontre, comme nous venons de le voir, que tel est bien le cas. Ses conclusions mettent ainsi un terme à un long débat sur l'étiologie de l'ostéomyélite aiguë que disputaient ses maîtres et ses contemporains.

Georg Albert Lücke (1829-1894), qui avait été, comme son élève Kocher, le disciple de Bernhard von Langenbeck (1810-1887) à Berlin, s'opposait en effet dans un article paru en $1874^{8}$, à la conception défendue par son propre maître à Berne, Hermann Demme (1802-1867). Selon ce dernier, l'ostéomyélite pouvait être causée par la conjonction de divers facteurs tels de petits traumatismes associés à une prédisposition individuelle momentanée ou durable: une maladie miasmatique, la fatigue ou de mauvaises conditions de vie $^{9}$. Lücke considérait par contre l'ostéomyélite comme une inflammation survenant dans les tissus profonds de l'organisme; elle présentait pour lui, tant sur le plan clinique que pathologique une analogie avec la pyémie. L'ostéomyélite devrait donc être de nature infectieuse ${ }^{10}$; elle devrait dépendre de deux facteurs: la présence d'un microorganisme pathogène et la qualité du terrain sur lequel peut se développer ce dernier ${ }^{11}$; il ne s'agissait cependant là que d'une hypothèse que seuls des expérimentations pourraient vérifier ${ }^{12}$.

Friedrich Julius Rosenbach (1842-1923), Professeur de chirurgie à Heidelberg, a cherché à déterminer en 1878 la nature de cet hypothétique agent infectieux ${ }^{13}$. Il a effectué à cet effet des expérimentations comparatives visant à évaluer l'effet d'un irritant chimique ou physique, ainsi que d'une 
préparation de pus contenant des microorganismes pathogènes, sur lesquels il n'a fourni cependant que peu de détails. Ces expérimentations le portaient à admettre que l'agent de l'ostéomyélite aiguë devrait être microbien. Ce dernier exercerait son action pathogène par voie sanguine. Le fait qu'il se localise électivement au niveau de la moelle osseuse impliquait pour Rosenbach qu'il devrait être spécifique de l'ostéomyélite ${ }^{14}$.

Une année plus tard, Kocher démontre expérimentalement, à l'inverse de ce dernier, que l'agent infectieux de l'ostéomyélite aiguë n'est autre que celui qui cause l'infection des plaies, c'est-à-dire un microorganisme non spécifique $^{15}$. Edwin Klebs (dans le laboratoire duquel Kocher a pratiqué) avait déjà étudié en 1872 un cas d'ostéomyélite, dans la moelle duquel il avait mis en évidence un microorganisme analogue à celui rencontré dans les plaies ${ }^{16}$. Il avait suggéré à ce moment-là que la porte d'entrée devait être cherchée au niveau des muqueuses ${ }^{17}$. Les recherches de Kocher font probablement écho à cette intuition ${ }^{18}$. Klebs évoquait également à ce moment-là l'idée que le microorganisme pathogène devrait trouver dans l'organisme infecté une «occasion» («eine Gelegenheit») de s'installer et de se développer ${ }^{19}$. Cette seconde intuition du pathologue bernois avait déjà inspiré Lücke ${ }^{20}$, qui parlait en 1874 d'un «punctum minoris resistentiae», c'est-à-dire d'un lieu où est survenu un trouble circulatoire local, d'un lieu qui présente, selon lui, les conditions favorables au développement d'un agent infectieux microbien ${ }^{21}$. Rosenbach a repris cette hypothèse en $1878^{22}$. Kocher déclare en 1879 être parvenu à la démontrer expérimentalement ${ }^{23}$.

\section{La strumite aiguë}

Fort de cette démonstration, Kocher se demande si les mêmes facteurs étiologiques peuvent expliquer, par analogie, la survenue d'une strumite aiguë, c'est-à-dire d'une inflammation du goitre, affection alors répandue dans la région bernoise ${ }^{24}$. Il publie en 1878 et en 1879 le résultat de ses recherches effectuées entre 1874 et 1878 chez ses propres patients ${ }^{25}$. Il rapporte ainsi qu'une infection digestive ou respiratoire a précédé dans 9 cas sur 24 le développement d'une strumite. Il observe de plus que cette affection survient avec prédilection si le goitre a subi au préalable un traumatisme ou a fait l'objet d'une ponction. L'hyperplasie de la thyrö̈de, la struma hyperplastica, constitue de même, par le biais des perturbations circulatoires et tissulaires qui s'y déroulent, une prédisposition à l'infection: 
«Acute Entzündung der Schilddrüse tritt in der Regel nur bei Vorhandensein einer localen Disposition auf.», écrit-il en 1878 «Diese Disposition besteht in anatomischen Veränderungen des Gewebes. Den gelindesten und häufigsten Grad einer Disposition in obigem Sinne bildet die Hyperplasie der Schilddrüse, die Struma hyperplastica, von dem Augenblicke an, wo in derselben Thromben, Blutergüsse oder regressive Metamorphosen, wie Verfettungen, Ablagerung von Colloidsubstanz stattfinden. Mit der Zunahme dieser secundären Veränderungen nimmt die Disposition zu und erreicht ihren höchsten Grad mit dem Zustandekommen von Gewebsnekrosen innerhalb des Strumagewebes ${ }^{26} . »$

Alors que son maître Lücke considérait encore en 1875 qu'un traumatisme était le plus souvent à l'origine du développement d'une strumite ${ }^{27}$, Kocher évoque le premier le caractère métastatique infectieux microbien de cette affection ${ }^{28}$. Il ne se fonde cependant que sur des observations cliniques: contrairement à ses recherches sur l'ostéomyélite, il ne fournit ici aucune démonstration expérimentale; la difficulté de mener à bien des expériences dans ce domaine en est probablement la cause.

\section{Une contribution originale à la théorie de l'infection}

L'intérêt des investigations de Kocher sur l'étiologie de l'ostéomyélite et de la strumite aiguës sera très tôt reconnu par l'un des plus grands pionniers de la recherche bactériologique des années 1880: Alexander Ogston (18441929), chirurgien écossais formé à Vienne, Prague et Berlin. Celui-ci démontre dès 1880, en se fondant sur les méthodes d'identification bactérienne qui venaient d'être mises au point par Robert Koch (1843-1910), que seuls les sphérobactéries du genre Micrococcus sont les agents responsables du développement des abcès ${ }^{29}$. Il considère cependant à la lumière des travaux de Kocher que la constitution individuelle, le terrain sur lequel se greffe le microorganisme, influence de manière significative l'intensité et la diffusion du processus infectieux: ce facteur peut déterminer la forme que prend la maladie.

«Ich kann nichts besseres thun», écrit-il en 1880, «als mit den Worten Kocher's zu schliessen: zwischen einer einfachen localisierten acuten Entzündung und den Fällen acutesten Pyämie besteht nur ein gradueller, ein quantitativer Unterschied ${ }^{30}$.»

Friedrich Julius Rosenbach démontre en 1884 le rôle étiologique du staphylocoque doré dans le développement de l'ostéomyélite aiguë ${ }^{31}$. Le vaudois Ernest Tavel (1858-1912) - un disciple de Koch ainsi qu'un proche collaborateur de Kocher au laboratoire de bactériologie de la clinique chirurgicale de 
Berne - démontre expérimentalement en 1892 l'étiologie bactérienne de la strumite aiguë ${ }^{32}$.

Ainsi, l'analyse des recherches de Kocher montre que ce dernier a apporté, à l'aube des années 1880 , une importante contribution à l'étude de l'étiologie des maladies infectieuses. Il a en premier lieu établi expérimentalement la nature infectieuse microbienne non spécifique de l'ostéomyélite aiguë, et a émis l'hypothèse que la strumite aiguë pourrait avoir la même cause. Il a en second lieu relevé - à la suite de ses maîtres Hermann Demme, Edwin Klebs et Georg Albert Lücke - le rôle du terrain dans le développement du phénomène infectieux. Le terrain correspond pour lui à la structure anatomo-pathologique du tissu contaminé.

Définir, comme il l'a fait en 1879, l'infection comme le produit d'une interaction entre un microorganisme et son hôte, peut être considéré comme original à une époque où l'enthousiasme des découvertes bactériologiques accordait un rôle prépondérant aux seuls agents microbiens ${ }^{33}$. Dès le début des années 1880, Rudolf Virchow a défendu l'idée qu'un facteur prédisposant d'ordre cellulaire pouvait être à l'origine d'une variation de la réponse de l'hôte à l'agent infectieux ${ }^{34}$. Un argument supplémentaire de poids venait ainsi alimenter la thèse de Kocher. Les travaux d'Otto Lubarsch (1860-1933), un disciple de Virchow, ont fécondé à la fin du $19^{\text {e }}$ siècle les recherches sur la nature des facteurs prédisposant à l'infection ${ }^{35}$. L'étude des mécanismes cellulaires et humoraux qui les déterminent font aujourd'hui l'objet de recherches multidisciplinaires associant les efforts des pathologues, des physiologistes, des infectiologues, des immunologues, ainsi que des généticiens. 
1 Kocher utilise des caustiques tels que l'ammoniaque ou l'huile de croton (arbuste de la famille des euphorbiacés dont les graines renferment une huile toxique); il emploie des extraits de cantharide (coléoptère vert doré long de $2 \mathrm{~cm}$ environ) comme substances vésicantes.

2 Kocher utilise une préparation effectuée par Marceli von Nencki (1847-1901), alors Professeur de chimie physiologique à Berne. Un pancréas frais de bœuf est placé sous vide dans une solution enrichie de sucre, de proteine et de gélatine. La putréfaction qui s'en suit sert de source de contamination lors de l'expérimentation. Le liquide putride contient des microcoques, c'est-à-dire des microorganismes à corps sphériques, ayant l'apparence de petites graines, groupés en grappe.

3 Kocher, Theodor, Zur Aetiologie der acuten Entzündungen, Archiv für klinische Chirurgie 23 (1879), 101-116.

4 Kocher, Theodor, Die acute Osteomyelitis, Deutsche Zeitschrift für Chirurgie 11 (1879), $87-251$

5 Billroth décrit pour la première fois la Coccobacteria septica en 1874 dans son ouvrage intitulé: Untersuchungen über die Vegetationsformen von Coccobacteria septica und den Antheil, welchen sie an der Entstehung und Verbreitung der accidentellen Wundkrankheiten haben. Billroth considère que la Coccobacteria septica est une algue qui donne naissance (selon les conditions physico-chimiques du milieu dans lequel elle se trouve) à diverses formes de microorganismes tels des microcoques, des streptocoques, des bactéries, des vibrions ainsi que des streptobactéries.

6 Kocher, Theodor, Die acute Osteomyelitis, Deutsche Zeitschrift für Chirurgie 11 (1879), 105.

7 Klebs, Edwin, Die Ursache der infectiösen Wundkrankheiten, Correspondenz-Blatt für Schweizer Ärzte 1 (1871), 241-246, et Klebs, Edwin, Beiträge zur pathologischen Anatomie der Schusswunden nach Beobachtungen in den Kriegslazarethen in Carlsruhe 1870 und 1871, Leipzig, 1872, 104-122. Klebs regroupe les diverses formes de microorganismes qu'il observe dans les plaies sous la dénomination de Microsporon septicum. Il s'agit pour lui d'un champignon polymorphe constitué d'un mycelium et de spores de tailles et de formes variables prenant à la fois l'aspect d'une bactérie et d'un champignon.

8 Lücke, Georg Albert, Die primäre infectiöse Knochenmark- und Knochenhautentzündung. Eine kritische Studie, Deutsche Zeitschrift für Chirurgie 4 (1874), 218-245, en particulier 218-220.

9 Demme, Hermann Askan, Zur Kenntniss und Behandlung der Osteomyelitis spontanea diffusa, Archiv für klinische Chirurgie 3 (1862), 169-271, en particulier 169-174 et 240-243.

10 Lücke, op. cit. (voir n. 8), p. 220.

11 Ibid. p. 240-241.

12 Ibid. p.241.

13 Rosenbach, Friedrich Julius, Beiträge zur Kenntniss der Osteomyelitis, Deutsche Zeitschrift für Chirurgie 10 (1878), 369-393 et 492-530.

14 Ibid. p. 392-393.

15 Kocher, Theodor, Zur Aetiologie der acuten Entzündungen, Archiv für klinische Chirurgie 23 (1879), 111-112.

16 Klebs 1872, op. cit. (voir n.7), p.119-120. Kocher a travaillé durant ses loisirs dans le 
laboratoire de Klebs à Berne: voir Tröhler, Ulrich, Der Nobelpreisträger Theodor Kocher 1841-1917, Basel 1984, p. 18.

17 Klebs 1872, op. cit., p. 120 : «Der Beginn dieser inneren Mycosen wird ... schwer festzustellen sein, und Sicheres kann über denselben bis jetzt nicht ausgesagt werden; nur darf mit einiger Wahrscheinlichkeit angenommen werden, dass die Importation der Keime durch die Schleimhäute, vielleicht vorzugsweise des Mundes und Rachens, geschieht. Die Entscheidung kann natürlich nur durch weitere klinische und experimentelle Untersuchungen geleistet werden.»

18 Kocher cite le texte de Klebs reproduit ci-dessus à la note 17, dans son article intitulé: Die acute Osteomyelitis, Deutsche Zeitschrift für Chirurgie 11 (1879), 105.

19 Klebs 1872, op. cit., p. 120 : ... «die einzelne Spore oder Bacterie wird wahrscheinlich lange mit der Blutmasse circuliren können, bevor sie Gelegenheit findet, sich irgendwo an der Gefässwand festzusetzen und sich weiter zu entwickeln.»

20 Lücke, op. cit. (voir n.8), p. 239-241.

21 Ibid., p. 241.

22 Rosenbach, op. cit. (voir n. 13), p. 392.

23 Kocher, Theodor, Zur Aetiologie der acuten Entzündungen, Archiv für klinische Chirurgie 23 (1879), 101.

24 Lücke confirme l'endémie goitreuse présente dans le canton de Berne dans l'ouvrage suivant: Die Krankheiten der Schilddrüse, Stuttgart 1875, p.31. Ce fait est également confirmé par Franz Merke dans son ouvrage intitulé: Geschichte und Ikonographie des endemischen Kropfes und Kretinismus, Bern, 1971, p. 237. L'ouvrage de Hermann Lebert, Die Krankheiten der Schilddrüse und ihre Behandlung, Breslau, 1862, p. 105-108, contient un très bon résumé des données historiques de l'époque sur le goitre. Les ouvrages généraux suivants sont également utiles à consulter: Bornhauser, Sigmund, Zur Geschichte der Schilddrüsen- und Kropfforschung im 19. Jahrhundert, Aarau, 1951, et Tröhler, op. cit. (voir n. 16), p. 121-162.

25 Kocher, Theodor, Zur Pathologie und Therapie des Kropfes, Deutsche Zeitschrift für Chirurgie 10 (1878), 191-229; Zur Aetiologie der acuten Entzündungen, Archiv für klinische Chirurgie 23 (1879), 112-115.

26 Kocher 1878, op. cit., p. 206.

27 Lücke 1875, op. cit. (voir n. 24), p. 87-92.

28 La thèse de Doctorat d' Eugen Stadelmann, intitulée: Beiträge zur Kenntnis und Kasuistik der Strumitis unter besonderer Berücksichtigung der Aetiologie, publiée à Freiburg i. Br. en 1912 est intéressante à ce propos : nous pouvons y lire dans l'introduction, qui fait l'état des connaissancs de l'époque, le passage suivant, p. 8 et 9: «Kocher war der erste, der mit Entschiedenheit die metastatische Entstehungsweise der Strumitis und ihren Zusammenhang mit vorgängigen Erkrankungen der Schilddrüse betonte [...].»

29 Ogston, Alexander, Über Abscesse, Archiv für klinische Chirurgie 25 (1880), 588-600. Ogston se réfère dans son introduction à l'ouvrage de Robert Koch de 1878, intitulé: Untersuchungen über die Aetiologie der Wundinfectionskrankheiten, Leipzig. Ce dernier fournit dans ce livre les bases méthodologiques (méthodes de culture et de colorations des bactéries) sur lesquelles Ogston se fonde dans la présente étude. Le microcoque appartient de ce fait pour Ogston à la famille des sphérobactéries. Pour plus de détails sur le problème de la classification des bactéries voir l'excellent ouvrage de William Bulloch, The History of Bacteriology, London 1938, p. 169-203. 
30 Ogston, op. cit., p. 599.

31 Rosenbach, Friedrich Julius, Mikro-Organismen bei den Wund-Infections-Krankheiten des Menschen, Wiesbaden, 1884, p.49-57, en particulier, p. 49.

32 Tavel, Ernest, Über die Aetiologie der Strumitis. Ein Beitrag zur Lehre von den haematogenen Infectionen, Basel 1892.

33 Voir Otto Lubarsch, Wandlungen in der Lehre von den Infektionskrankheiten, Deutsche Zeitschrift für Chirurgie 234 (1931), 32, qui donne une bonne vue d'ensemble sur la question.

34 Virchow, Rudolf, Krankheitswesen und Krankheitsursachen, Archiv für pathologische Anatomie und Physiologie und für klinische Medicin 79 (1880), 1-19 et 185-228. Cet article est en fait une réponse à Edwin Klebs qui soutenait en 1878 à l'encontre de la théorie cellulaire que seul, un agent microbien est responsable du développement d'une maladie infectieuse. Virchow défend ici au contraire l'idée que la maladie infectieuse est le produit d'une interaction active entre le microorganisme et la cellule (p. 210-212). Il appelle la participation cellulaire «innere Ursache» ou «Praedispositio». Voir également à ce propos l'article de Johannes Orth, Rudolf Virchow und die Bacteriologie, Deutsche medizinische Wochenschrift 36 (1910), 1937-1939.

35 Voir Lubarsch, op. cit. (voir n. 33), p. 32-38. 


\section{Summary}

\section{Theodor Kocher's research on the aetiology of acute osteomyelitis and strumitis}

In 1879, Kocher demonstrated experimentally that osteomyelitis is an infectious disease which is caused by a non-specific microorganism (the "micrococcus"). He showed that the infection of the bone marrow is the consequence of a hematogenous dissemination of a local infection, either cutaneous or mucous. The clinical and pathological form of the disease depends not only on the virulence of the microorganism, but also on the state of the tissue in which it develops. In the same year, Kocher suggested that acute strumitis (inflammation of the goitre) has the same aetiology; he did not, however, produce any experimental evidence for this. Thus, Kocher defined in an original way infection as the result of an interaction between a microorganism and his host.

\section{Zusammenfassung}

\section{Theodor Kochers Forschungen über die Aetiologie der akuten Osteomyelitis und Strumitis}

Kocher hat 1879 durch Experimente bewiesen, dass die akute Osteomyelitis eine mikrobielle Infektionskrankheit ist. Als Erreger betrachtete er einen unspezifischen Mikroorganismus, den Mikrococcus. Er zeigte, dass die Knochenmarksinfektion die Folge einer lokalen Haut- oder Schleimhautinfektion ist, die durch den Blutstrom verbreitet wird. Das klinische und pathologische Bild der Krankheit hängt nach Kocher nicht nur von der Virulenz des Erregers ab, sondern ebensosehr von der Beschaffenheit des Gewebes, auf dem er sich entwickelt. Im gleichen Jahr sprach er die Vorstellung aus, dass die akute Strumitis (die Entzündung des Kropfes) die gleiche Ursache haben könnte; allerdings hat er dies nicht experimentell nachgewiesen. Wir können somit festhalten, dass Kocher gegen 1880 selbständig festgestellt hat, dass die Infektion das Ergebnis des Zusammenwirkens eines Erregers und seines Wirtes ist.

Lazare Benaroyo, Dr. med.

Medizinhistorisches Institut und Museum

der Universität Zürich

Rämistrasse 71

8006 Zürich 